Ciencia y Educación, Vol. 5, No. 1, enero-abril, 2021

ISSN (impreso): 2613-8794・ISSN (en línea): 2613-8808

DOI: https://doi.org/10.22206/cyed.2021.v5i1.pp7-24

\title{
Actitudes de los maestros ante la inclusión educativa en Colombia, Guatemala y España
}

\author{
Teachers' attitudes towards educational inclusion in \\ Colombia, Guatemala and Spain
}

Laura Hernández Izquierdo ${ }^{a}$ ORCID: 0000-0003-0434-679X
Álvaro Marchesi ${ }^{\mathrm{b}}$ ORCID: 0000-0001-9429-7674

Recibido: $16 / 04 / 2020 \bullet$ Aprobado: 3/07/2020

Cómo citar: Hernández Izquierdo, L., \& Marchesi, Álvaro. (2021). Actitudes de los maestros ante la inclusión educativa en Colombia, Guatemala y España. Ciencia y Educación, 5(1), 7-24. https://doi.org/10.22206/cyed.2021.v5i1.pp7-24

Resumen

Los maestros son un elemento clave en el desarrollo de la educación inclusiva y sus actitudes, a su vez, repercuten de manera directa en las actitudes de sus propios alumnos. Por ello, es fundamental conocer la opinión del profesorado hacia la diversidad y la permanencia de diferentes alumnos en sus aulas. Para este estudio se contó con la colaboración de 132 maestros y 40 especialistas en educación de Guatemala, Colombia y España, a los que se les aplicó un cuestionario individual además de entrevistas abiertas. Profesores de tres países con diferente desarrollo social, cultural y educativo transmitieron sus impresiones sobre la inclusión de alumnos con discapacidad y la inclusión de alumnos de otras culturas. Entre otros, los resultados muestran como en las tres naciones no existen las condiciones adecuadas para conseguir una inclusión plena de los alumnos con necesidades educativas especiales en la escuela ordinaria. Por otro lado, se identifican barreras actitudinales y de discriminación hacia la población indígena y afrodescendiente en

\begin{abstract}
Teachers are a key element in the development of inclusive education and their attitudes have a direct impact on the attitudes of their own students. Therefore, it is essential to know the opinion of teachers towards in-class teaching and diversity. For this reason, 132 teachers and 40 educational specialists from Guatemala, Colombia and Spain participated in this research by answering individual questionnaires and conducting open interviews respectively. The participating teachers, coming from three countries with different social, cultural and educational backgrounds, shared their opinions about the inclusion of students with disabilities and the inclusion of students from other cultures. The results show how in the aforementioned three countries, the adequate conditions to achieve the full inclusion of students with special educational needs in mainstream schools are not yet present. Attitudinal barriers and discrimination towards the indigenous and Afro-descendant population in Guatemala and Colombia respectively,
\end{abstract}

a Universidad Complutense Madrid, España. Correo-e: laurhern@ucm.es

b Universidad Complutense Madrid, España. Correo-e: amarches@psi.ucm.es 
Guatemala y Colombia, respectivamente, que son transmitidas a los más pequeños desde su contexto educativo, familiar y social.

Palabras clave: América Latina; educación; diversidad; cultura; transformación social; políticas educacionales; docentes.

\section{Introducción}

Un indicativo del crecimiento de una cultura de inclusión es la existencia de un consenso en torno a los valores de respeto por la diferencia y un compromiso de ofrecer a todos los estudiantes el acceso a las oportunidades de aprendizaje (Ainscow, 2017).

Los docentes son considerados como elementos claves en el desarrollo de actitudes positivas hacia los alumnos en la implementación de la educación inclusiva (Meijer, 2003). Se hace imprescindible que el docente muestre actitudes positivas que favorezcan la inclusión de manera general.

En este marco, el presente artículo se focaliza en la comparación de las actitudes de los maestros de tres países (Colombia, España y Guatemala) con relación a la inclusión del alumnado culturalmente minoritario y del alumnado con necesidades educativas especiales.

Tras presentar un marco teórico sobre las actitudes de los docentes hacia la inclusión y los alumnos con necesidades educativas especiales y hacia la diversidad cultural, este artículo presenta la realidad sociocultural y educativa de los tres países implicados, poniendo el foco en el colectivo cultural estudiado en cada uno de ellos (afrodescendientes en Colombia, población indígena en Guatemala y alumnos inmigrantes en España).

Para el desarrollo del estudio se utilizaron técnicas mixtas; cuantitativa (cuestionarios) y cualitativa (entrevista). Con el fin de facilitar la interpretación de los datos, estos se presentan interconectando los datos cuantitativos y cualitativos, organizados en cada una which are transmitted to the youngest from their educational, family and social context, are also identified.

Keywords: Latin America; Education; Diversity; Culture; social transformation, educational policies, teachers.

de las cuatro hipótesis establecidas (dos de ellas vinculadas a las actitudes de los maestros hacia los alumnos con necesidades educativas especiales y las dos en las actitudes de los maestros hacia los alumnos de otras culturas).

\section{Marco teórico}

\subsection{Actitudes de los profesores hacia los alumnos con necesidades educativas especiales y la educación inclusiva}

Uno de los factores de mayor influencia en el desarrollo de modelos inclusivos en las escuelas es el de las actitudes y expectativas que el profesorado muestra ante la diversidad de sus alumnos. Su influencia en la autoestima, la motivación y el aprendizaje ha sido ampliamente comprobada (Sales et al., 2001). El conocimiento de las actitudes de los profesores hacia la diversidad de los alumnos y hacia la inclusión en sus aulas es fundamental para impulsar proyectos de cambio.

Echeita (2017), entre otros, establece como principios y valores propios de los docentes que comparten la pedagogía inclusiva; ser un profesorado reflexivo que continuamente establece relación entre lo que hace y cómo lo hace, con los valores propios de una educación inclusiva (equidad, respeto a la diversidad, justicia, igualdad, etc.).

Estos principios o creencias de los docentes sobre el aprendizaje, sobre si las capacidades del alumno son estables o maleables a lo largo del tiempo y sobre el grado de influencia que puede tener la educación en el progreso de los alumnos afectan a las actitudes del profesor, a sus expectativas y a su forma de orientar 
su enseñanza (Marchesi y Martín, 2014). Cuando un maestro tiene respecto a un alumno concreto una elevada expectativa de éxito, tiende a transmitírsela de forma implícita, demostrándole confianza en sus posibilidades. Así, por ejemplo, un maestro cuyas actitudes sean negativas hacia la inclusión de los alumnos de culturas minoritarias o de niños con necesidades educativas especiales, tenderá a bajar sus expectativas escolares hacia estos niños. El afecto, la compasión y la sensibilidad hacia los alumnos con más dificultades contribuyen a que el profesor se preocupe más por ellos y haga un esfuerzo suplementario para aprovechar todas las oportunidades posibles que favorezcan su interés y su participación en actividades de aprendizaje (Marchesi, 2017).

Varios estudios han tratado de comprobar las actitudes de los profesores hacia los alumnos con necesidades educativas especiales y la educación inclusiva. Algunos de ellos afirmaron que los maestros son positivos hacia la filosofía general (Avramidis y Norwich, 2002; Marshall, et al., 2002; Abbott, 2006), mientras que otros han comprobado que los profesores tienen serias reservas acerca de la educación inclusiva en la práctica (Florian, 2014; Pearman et al., 1997).

Boer (2012) realizó una revisión de 26 estudios que habían analizado la cuestión y comprobó que la mayoría de los docentes tienen actitudes neutras o negativas hacia la inclusión de alumnos con necesidades educativas especiales en la educación primaria regular. Esto puede ser debido no a una actitud negativa, sino a no saber cómo dirigirse hacia estos alumnos, por desconocimiento.

La falta de formación adecuada podría ocasionar desinterés y un abierto rechazo hacia la incorporación en las aulas ordinarias de alumnos con determinadas necesidades o con desventajas sociales o culturales. Por otro lado, las investigaciones han demostrado resultados positivos de la formación del profesorado en materia de educación inclusiva (Rea et al., 2011). Esto indica que la mejora del conocimiento de los maestros acerca de la diversidad puede ser un primer paso para cambiar su actitud general. Este aumento de conocimiento posteriormente puede conducir a un cambio en los sentimientos de los maestros y, en última instancia, a modificar su comportamiento real en el aula.

\subsection{Actitudes de los profesores hacia la diversidad cultural}

La atención a la diversidad cultural ha estado en el centro del debate sobre la adecuación o reformulación de propuestas educativas en diferentes países, motivados por la preocupación de conciliar la cohesión social con las diferencias culturales (Fernández y Terren, 2008).

Las diferencias étnicas plantean también un reto importante a la educación en aquellos países en los que habitan diferentes grupos culturales. La convivencia en un contexto educativo con diversidad cultural, con un alumnado heterogéneo, con diferentes intereses y motivaciones; constituye todo un reto para el profesorado (Carbonell, 2002). Aunque el propio concepto de cultura incluye la diversidad, pues describe las normas, valores, símbolos, productos y tradiciones que han desarrollado la variedad de grupos humanos a lo largo de la historia, su significado se sitúa en otra dimensión cuando los diferentes grupos culturales conviven en un mismo territorio. Es entonces cuando las relaciones entre ellos, la dominación o sumisión de unos frente a otros, y las posibilidades de progreso a lo largo de la vida tienen un especial impacto en la vida social y educativa.

En ocasiones, las diferencias culturales son sinónimo de desigualdad. En Latinoamérica, como señala Calderón (1996), la secular negación del otro es parte esencial de la historia de la colonización que se prolonga bajo formas de discriminación y exclusión a lo largo de la vida. Existen, pues, culturas y ciudadanos de primera y de segunda categoría, en donde las personas blancas que además son poseedora de los mayores recursos económicos ocupan el nivel más alto en esta jerarquía clasista.

Estos modelos de relación profundamente enraizados en las creencias sociales, más allá de los discursos públicos que defienden la igualdad y la superación de la discriminación, actúan de forma potente, y en 
muchas ocasiones, de manera poco consciente en las relaciones que se generan en la comunidad educativa. Su influencia se manifiesta en los estereotipos sociales, en las expectativas hacia los alumnos, en las condiciones de estudio, en el apoyo familiar y en la interiorización de los alumnos de sus posibilidades para seguir estudiando.

López (2012) analiza la equidad educativa y diversidad cultural en América Latina, indicando que la escuela y sus docentes no solo no saben tratar con alumnos diferentes culturalmente, sino que además llegan a estigmatizarlo y juzgarlo por su origen, su apariencia o sus costumbres. Hay que tener en cuenta que el nivel de vida y las posibilidades de progreso de los grupos culturales minoritarios en los países latinoamericanos son muy inferiores a aquel que se sitúa en el vértice superior de la pirámide social.

Existe, pues, un reto enorme para romper posibles estereotipos profundamente arraigados y generar una dinámica social y educativa que eleve las expectativas de los profesores hacia los alumnos de aquellas culturas menos valoradas. Por ello, el estudio de las actitudes de los profesores hacia los grupos culturales menos valorados es tan importante, ya que orienta los procesos necesarios de cambio.

Igualmente, es preciso que los maestros conozcan las condiciones en las que viven los alumnos de las culturas minoritarias, compartan o sintonicen con sus raíces étnicas y lingüísticas y sean capaces de conseguir que sus alumnos valoren sus aprendizajes. Es imprescindible que los docentes faciliten a todos los alumnos oportunidades de reconocimiento y valoración de sus identidades, de sus herencias culturales y familiares (Echeita, 2017).

Es necesario promover una formación de los docentes respetuosa con las minorías para poder trabajar actitudes positivas hacia ellas, en el reconocimiento y aceptación de otras culturas diferentes con sus propios valores y significados (Olivencia, 2008). Aprender a enseñar en contextos culturalmente diversos, en un plano afectivo y reflexivo crítico, es una meta necesaria de alcanzar para todos los maestros, independientemente de su origen étnico o cultural, o el contexto donde desarrolla su labor educativa, cuya principal perspectiva es una formación de calidad (Zapata, 2014).

El incremento de las expectativas de los profesores, que debe también trasladarse a las familias para que apoyen los estudios de sus hijos, así como la autovaloración y la confianza de los propios alumnos son factores necesarios para conseguir un mejor aprendizaje.

\subsection{Contexto social y educativo en Colombia, Guatemala y Espańa}

2.3.1. COLOMBIA: Contexto social y educativo: población afrodescendiente y alumnos con necesidades educativas especiales asociadas a discapacidad

Colombia posee una población multicultural, que es, en su mayoría, resultado del mestizaje entre europeos, indígenas y africanos. Durante el último censo realizado en el país en 2018, se reportó que los afrocolombianos representan el $6.75 \%$ de la población, casi 3 millones de personas, según una proyección del Departamento Administrativo Nacional de Estadística.

El Estado ha realizado esfuerzos significativos en materia de política pública orientada al reconocimiento de los derechos étnicos y territoriales y ha implementado numerosos planes y proyectos nacionales, regionales y municipales con el objetivo de superar la situación de pobreza, marginación y exclusión en la que vive esta población. Existe un amplio marco legislativo que no solamente reconoce la diversidad étnica y cultural del país, sino también los derechos de los afrodescendientes a poseer los territorios de ocupación ancestral, a no ser discriminados, a la igualdad en el acceso a oportunidades, a participar en los organismos de planeación y decisión y a participar en política.

A pesar de la copiosa normativa existente y de la gran cantidad de programas implementados para mejorar su calidad de vida, existen dificultades en 
materia de inclusión para la población afrodescendiente. La misma, exhibe las condiciones de vida más precarias. Roux (2010) denuncia la situación social de la población afrocolombiana, entre las que se indica que el porcentaje de afrocolombianos que está por debajo de la línea de pobreza es superior al de los "no étnicos",

desigualdad en el acceso al mercado laboral y su vinculación con trabajos de baja calidad, carencia en la ingesta de alimentos básicos y desnutrición, altas tasas de analfabetismo y dificultades en el acceso a la educación, tasas más bajas de acceso a la educación superior, carencia de una etnoeducación, precarias condiciones de salud, déficit de vivienda y menor esperanza de vida al nacer:

Asimismo, la Comisión Intersectorial ${ }^{a}$ identifica barreras existentes en la sociedad colombiana hacia las personas afrodescendientes (Roux, 2010), como racismo y discriminación racial, baja participación y representación en espacios políticos e institucionales de decisión, escaso reconocimiento y valoración de la diversidad étnica y cultural, baja disponibilidad de información sobre la población afrodescendiente (que limita la focalización y la definición de una política pública ajustada a las particularidades étnicas y territoriales), débil capacidad institucional de los procesos organizativos, deficiencias en materia de seguridad jurídica de los derechos de propiedad de los territorios colectivos, o acceso limitado a programas de subsidio.

Respecto a la población con discapacidad, el informe "Discapacidad en Colombia: reto para la Inclusión en Capital Humano" (Gómez Beltrán, 2010) denuncia que el $48,5 \%$ de las personas percibe barreras en la vía pública, el 34,4 \% de las personas se enfrenta a barreras al hacer uso del trasporte público, por lo que tienen grandes dificultades para asistir a sus servicios de salud, educación, rehabilitación y trabajo; mientras que el $12,7 \%$ de las personas registradas encuentra barreas físicas en los centros educativos; y el 16,5\% tiene barreras en los lugares de trabajo.
2.3.2 GUATEMALA: contexto social y educativo; población indígenay alumnos con necesidades educativas especiales asociadas a discapacidad

Guatemala es un país multiétnico, multilingüe y pluricultural, en el que cohabitan tres pueblos indígenas (maya, xinca y garífuna) y el pueblo ladino (mestizo, blanco y criollo) en el que se reconoce la existencia de 22 idiomas mayas, el garífuna, el xinca y el español.

La importancia histórica del "Acuerdo de Identidad y Derechos de los Pueblos Indígenas" permite reconocer un antes y un después en la lucha de los pueblos indígenas por ser reconocidos como ciudadanos de pleno derecho, en la imperiosa necesidad de la reforma de Estado con enfoque intercultural y en la construcción de la nación con capacidades multiculturales.

La mayoría de la población indígena de Guatemala es hablante de alguno de los 22 idiomas pertenecientes a la familia lingüística maya. No obstante, el castellano es la lengua franca utilizada para la realización de las diversas actividades productivas en la múltiple vida social y política. Quienes no lo hablan están en una gran desventaja comunicativa interétnica.

Actualmente existen graves problemas sociales y económicos que afectan a gran parte de la población indígena con relación al acceso a la tierra, derechos sociales y laborales, servicios públicos y el reconocimiento efectivo de la administración de justicia indígena. Durante siglos, en la época colonial y en la independiente, los pueblos indígenas de Guatemala no han sido igualmente incluidos en el proceso de desarrollo, y no es sino hasta época reciente, que el reconocimiento de sus derechos políticos, entre ellos el goce pleno de su ciudadanía, ha dado oportunidad para iniciar su incorporación a las diversas instancias de la vida nacional. No obstante, en su mayoría, sus integrantes ocupan los estratos más bajos de la estructura social y la raíz colonial de la dominación que se mantiene, los hace víctimas de racismo y discriminación.

a. Institución creada con el fin de hacer más efectivas las políticas públicas a favor de la población afrocolombiana, mediante el Decreto 4181 de 2007. 
La educación bilingüe responde a las características, necesidades e intereses de los alumnos indígenas, en lugares conformados por diversos grupos étnicos y lingüísticos. La misma, se lleva a cabo a través de programas en los subsistemas de educación escolar y educación extraescolar o paralela. Esta se realiza para afirmar y fortalecer la identidad y los valores culturales de las comunidades lingüísticas. Cabe señalar que la cobertura de la educación bilingüe es baja en un país cuya población indígena es mayoritaria. No obstante, a lo largo de los últimos años ha habido aumentos considerables en los esfuerzos del Ministerio de Educación con relación a la educación intercultural bilingüe.

En lo que refiere a la situación educativa y social de las personas con discapacidad, hay que destacar la falta de información en el momento de desarrollo de estudio, lo que refleja que estas personas no cuentan con condiciones adecuadas para su atención debido a un limitado acceso a la educación, la salud y la vida comunitaria y cultural.

El Consejo Nacional para la Atención de las Personas con Discapacidad (CONADI, 2006) caracterizó la situación social de las personas con discapacidad en Guatemala de acuerdo con los siguientes indicadores: servicios especializados escasos y centralizados, desconocimiento de las personas con discapacidad de instituciones que prestan servicios (lo que dificulta la detección temprana), ingresos escasos para acceder a servicios especializados, debilidad de las organizaciones de y para personas con discapacidad, finalmente, invisibilidad del tema en las políticas sociales sectoriales.

\subsubsection{ESPAÑA. Contexto social y educativo: población inmigrante y con alumnos con necesi- dades educativas especiales asociadas a discapa- cidad}

Lo característico de la inmigración en España fue la celeridad con la que se produjo la entrada de inmi- grantes entre el año 2000 y 2006. En efecto, pasó de ser uno de los países de la OCDE con menor porcentaje de extranjeros en el año 2000, a situarse con un porcentaje de extranjeros superior a la media de la OCDE y con una notable pluralidad que lo diferencia del resto de los países de su entorno (Rahona y Morales, 2013). Las últimas estadísticas publicadas del Ministerio de Educación reflejan, en el curso 2017-2018, la incorporación de 24,458 alumnos procedentes de otros sistemas educativos.

En relación con la llegada de inmigrantes, una de las políticas sociales que se ve más afectada en España es la política educativa. La educación es una de las principales instancias para facilitar y garantizar la integración y socialización de los extranjeros, ya que facilita el aprendizaje y asimilación de la historia y cultura del país de destino y, sobre todo, del idioma, desconocido en algunos casos cuando los jóvenes se integran a la escuela. La inclusión de alumnos procedentes de otros países o culturas se dificulta en muchas ocasiones por la edad a la que ingresan al sistema escolar espańol, por su trayectoria educativa previa y por las dificultades económicas y sociales de la familia, que pueden ser determinantes en el abandono prematuro de los estudios.

El desigual reparto entre los dos tipos de centros financiados públicamente es una de las principales dificultades a la hora de garantizar la integración en las escuelas de los niños y jóvenes inmigrantes, ya que su agrupación en determinados centros puede derivar en la existencia de "centros-gueto", en su mayoría de titularidad pública, en los que se concentra un número excesivo de estudiantes extranjeros.

La educación de alumnos con necesidades educativas especiales en España se ha evolucionado de sistemas segregadores y diferenciados, a la inclusión de los alumnos en escuelas ordinarias. En el curso 2017-2018, el porcentaje de alumnado con necesidades educativas especiales que recibe apoyo supone el 2,7\% del alumnado matriculado en las enseñanzas no universitarias (Ministerio de Educación y Formación Profesional, 2018). 


\section{Metodología}

\section{1. Objetivo}

El objetivo fue contrastar las opiniones de los maestros de Colombia, España y Guatemala, de la etapa de educación infantil y primaria (alumnos entre los 3 y 12 años), sobre el proceso de inclusión de alumnos con diferentes culturas y de alumnos con necesidades educativas especiales asociadas a discapacidad.

\subsection{Hipótesis}

- Hipótesis 1. Los maestros valoran positivamente la inclusión de los alumnos con necesidades educativas especiales asociadas a discapacidad. Los maestros que trabajan en países con menos recursos (Colombia y Guatemala) mostrarán más reservas que los españoles.

- Hipótesis 2. Los maestros consideran que los alumnos con necesidades educativas especiales asociadas a discapacidad tienen dificultades de relación con su grupo de iguales.

- Hipótesis 3. Los maestros valoran positivamente la inclusión de los alumnos de otras culturas. Los países con mayor jerarquía cultural (Colombia y Guatemala) mostrarán menos apoyo.

- Hipótesis 4. Los maestros opinan que los alumnos de otras culturas no tienen dificultades en las relaciones sociales.

\subsection{Participantes}

Los participantes fueron:

132 maestros (22 maestros de España, 30 maestros de Colombia, 39 maestros de Guatemala de estudiantes indígenas, 42 maestros de Guatemala de estudiantes ladinos).
- 40 especialistas en educación (8 en Colombia, 22 en Guatemala y 10 en España). Entre ellos se encontraban directores de escuelas, profesores universitarios, líderes comunitarios y investigadores en educación.

\subsection{Técnicas de recolección de información}

Para el desarrollo del estudio se utilizaron técnicas mixtas; cuantitativa (cuestionarios) y cualitativa (entrevista). El cuestionario estaba compuesto por 21 ítems tipo Likert de respuesta múltiple (totalmente en desacuerdo, en desacuerdo, de acuerdo, muy de acuerdo). De los 21 ítems, tres eran afirmaciones sobre el concepto de inclusión, nueve ítems se relacionaban con las actitudes hacia los niños con discapacidad y nueve ítems se vinculaban a las actitudes hacia los niños de otras culturas.

Para la elaboración del cuestionario, se tomó de referencia el cuestionario elaborado con el estudio "Inclusion: a question of attitudes?" (Boer Anke, 2012). El cuestionario de los docentes de Boer incluye 19 ítems que miden las actitudes hacia la discapacidad. El análisis de la escala de Boer (2012) obtuvo coeficientes satisfactorios de validez $(\mathrm{H}=0.48, \mathrm{H}=0.40$, $\mathrm{H}=0,50$, respectivamente) y coeficientes altos de fiabilidad $(\rho=0.91, \rho=0,92, y \rho=0,92$, respectivamente) por lo que se utilizó en el estudio realizando los siguientes ajustes:

1. Incorporación de la diversidad cultural como nueva variable de estudio.

2. Disminución de los ítems vinculados a las actitudes hacia los alumnos con necesidades educativas especiales asociadas a discapacidad.

La validación del cuestionario elaborado se realizó a través de una sesión de debate y/o reflexión con diez docentes, en la que se intercambiaron ideas y propuestas de ajustes al cuestionario a través de la técnica de

b. En el caso de Guatemala se diferencian dos grupos de maestros: por un lado, maestros de comunidades indígenas cuyos alumnos son principalmente indígenas, y por otro, maestros de zonas urbanas y ladinas cuyos alumnos son mestizos y/o blancos. La decisión de incorporar un análisis diferenciado de estos dos grupos fue que al realizar el estudio diferencial entre los países, solo en Guatemala se encontraron diferencias entre los maestros que daban clase a diferentes colectivos. 
brainstorming o "lluvia de ideas". El número y características de los ítems se mantuvieron como estaban en la versión inicial, pero se matizó la redacción de determinados ítems. Una vez cerrado el cuestionario, se facilitó el borrador a especialista de Guatemala y Colombia para realizar el ajuste idiomático necesario para adaptarlo al contexto cultural de cada país.

Los cuestionarios fueron aplicados a los 132 maestros de manera individual en cada una de las escuelas visitadas en los tres países (27 escuelas en total: 7 en Colombia, 14 escuelas en Guatemala y 6 en España). Su aplicación fue de 15-20 minutos aproximadamente. Se encontraron diferentes niveles de formación de los maestros, pero en ningún caso se detectaron graves problemas de comprensión de los ítems.

Así mismo, se realizaron 40 entrevistas abiertas ( 8 en Colombia, 22 en Guatemala y 10 en España) a diferentes personas que podían aportar información relevante para la posterior interpretación de los datos. Las entrevistas se basaban en conversaciones distendidas sobre los diferentes temas vinculados al estudio: situación educativa del país, diversidad, intervención educativa con diferentes grupos étnicos o culturales e intervención educativa con alumnos con necesidades educativas especiales asociadas. Las entrevistas fueron grabadas (solo con material de audio) y posteriormente analizadas y clasificado su contenido.

Por lo que se refiere al análisis de las respuestas de los cuestionarios, se tuvo en cuenta el análisis en función del grupo cultural y del país. Se utilizó análisis de frecuencia ya que en las preguntas se pedía el grado de acuerdo con las afirmaciones presentadas.

\subsection{Procedimiento}

El estudio se llevó a cabo en Colombia, Guatemala y en España. La selección de los países implicados se basó en el criterio del acceso a diferentes grupos sociales: Colombia por poseer un alto porcentaje de población afrodescendiente, Guatemala por su alto porcentaje de población indígena y España por la incorporación a sus aulas de un alto número de alumnos inmigrantes.
El contacto con las escuelas se realizó en los tres países atendiendo a los siguientes parámetros:

1. Escuelas que pertenecian a localidades con niveles socioeconómicos diferentes, con el objetivo de tener acceso a escuelas con docentes de las diferentes clases sociales (clase alta, media y baja).

2. Escuelas con diferente titularidad (escuelas públicas $y$ privadas). El criterio anterior implicaba la necesidad de acudir tanto a escuelas públicas como privadas.

3. Presencia de alumnado de diferentes grupos culturales:

- En Colombia se identificaron escuelas con y sin alumnos afrodescendientes.

- En Guatemala se identificaron escuelas con y sin alumnos indígenas.

- En el caso de Espańa se identificaron escuelas con y sin alumnos inmigrantes.

En un inicio se intentó incluir entre los criterios de selección de las escuelas de los tres países, el hecho de que se tratara de centros con y sin alumnos con necesidades educativas especiales asociadas a condiciones de discapacidad. En el proceso de identificación de estas, se encontró dificultad en Colombia para acceder a escuelas que escolarizaran estos alumnos. Ello obligo a que dicha variable (presencia o no de alumnos con discapacidad) no fuera controlada y que el acceso a escuelas al respecto fuera realizado al azar.

\section{Resultados}

\subsubsection{Hipótesis 1. "Los maestros valoran positi- vamente la inclusión de los alumnos con necesidades educativas especiales asociadas a discapacidad"}

El 52,6 \% de los maestros encuestados en los tres países opinó que los alumnos con necesidades educativas especiales asociadas a discapacidad aprenden más si asisten a escuelas ordinarias. El 47,4 \% no valoró favorablemente su inclusión educativa. 


\section{Figura 1}

Porcentaje de maestros que considera que "los alumnos con necesidades educativas especiales asociadas a discapacidad aprenden más si asisten a escuelas normales"

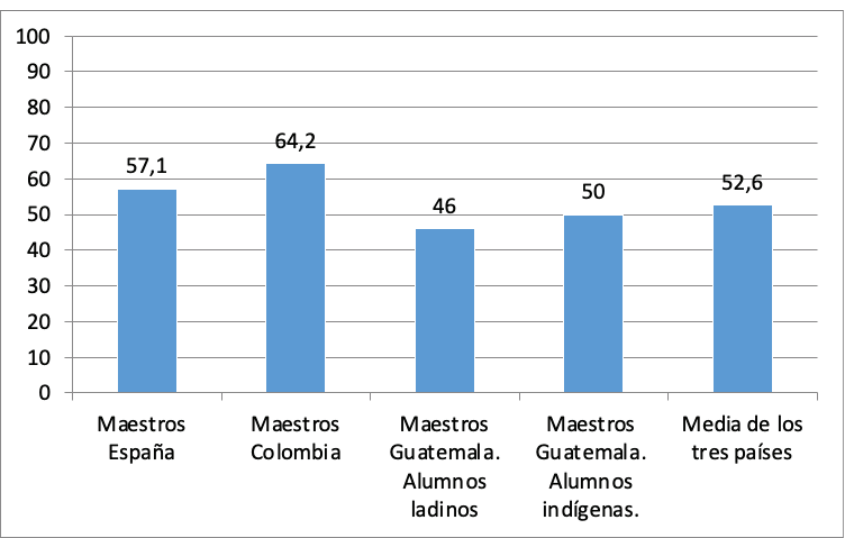

Más de la mitad de los maestros de escuelas de Guatemala con alumnos ladinos e indígenas (54\% y $50 \%$, respectivamente), consideran que los alumnos con discapacidad no aprenden más si asisten a escuelas normales.

En las entrevistas realizadas en Guatemala, entre las necesidades detectadas para la incorporación de los niños con discapacidad en las aulas, se destaca la necesidad de formación, inicial y permanente que otorgue a los maestros las herramientas necesarias para lograr una inclusión de calidad y conseguir que el proceso de enseńanza y aprendizaje se realice con éxito.

"Desde el punto de vista de la inclusión de alumnos con discapacidad, se necesitaría mucha formación tanto inicial como en los maestros en servicio. Los maestros no están formados. No están en condiciones para realizar las adecuaciones curriculares. Se les dice que deben hacerlas, pero no saben. Es fundamental la formación para que puedan encontrar la forma de desarrollar en el aula una metodología adecuada”. (Especialista en educación inclusiva. Guatemala).

"Es necesaria la inclusión, no podemos separarlos, pero siempre y cuando se cuente con los medios y recursos. Decirle al maestro que haga las adecuaciones no es sencillo. Al final se dice que los niños con discapacidad están en el aula y que están incluidos, pero algunos maestros solo los entretienen. Socialmente participan, pero no desarrollan todas sus capacidades y habilidades. Si los maestros estuvieran preparados, podrían realizar las adecuaciones necesarias." (Especialista en primera infancia y educación inclusiva. Guatemala).

"Es poca la capacitación que se puede dar desde educación especial porque son muchas escuelas y muy distantes. Hay que sensibilizar a los maestros, supervisores, padres y directores. Es fundamental. Hay problema en la formación inicial de los maestros. No saben metodologías" (Coordinadora programa de educación especial. Ministerio de Educación, Guatemala).

Esta falta de formación del profesorado genera en muchas ocasiones miedo, resistencia e inseguridad en los maestros que les conduce a no tener las actitudes favorables para atender a los alumnos con discapacidad.

"Lo que más les cuesta es perder el miedo, porque la mayoría, como no están acostumbrados, tienen miedos. Hacen muchas preguntas. Muchos de los profesores tienen miedo y no reportan que tienen dificultades. No saben qué hacer con los alumnos. El sistema solo les enseña a trabajar con alumnos regulares." (Coordinadora de educación especial. Ministerio de Educación, Guatemala).

Inicialmente, se esperaba en el estudio que los maestros de países con menos recursos (Colombia y Guatemala) mostraran más reservas a la inclusión de alumnos con necesidades educativas especiales asociadas a discapacidad, que los maestros de España. Sin embargo, las diferencias son escasas entre los maestros guatemaltecos y los maestros españoles, lo que indica una creencia bastante extendida de que la inclusión no siempre es positiva para los alumnos con necesidades educativas especiales asociadas a discapacidad. Es posible también pensar que los maestros se manifestaran recelosos, al no diferenciarse en la pregunta a los alumnos con discapacidad más profunda, de aquellos otros con dificultades leves y moderadas. 
Así mismo, el 64,2 \% de los maestros colombianos encuestados consideró que los alumnos con necesidades educativas especiales aprenden más en escuelas ordinarias; este porcentaje es superior a los resultados de los maestros españoles, quienes lo consideran un 51,7 \%. No obstante, es necesario especificar que en el caso de Colombia, solo se tuvo acceso a una escuela en la que estudiaba una niña con discapacidad motora, lo que puede influir en unos resultados más positivos. Las respuestas pueden ser más negativas en aquellos maestros que han tenido dificultades con alumnos con discapacidad a lo largo de su trayectoria profesional.

Entre los testimonios obtenidos en España y Colombia sobre la inclusión de alumnos con necesidades educativas especiales se destaca:

"Trabajar con niños con necesidades es más difícil, pero se consigue si hay disposición" (Maestro de España).

"Con los niños con necesidades nos cuesta más. Es más complejo" (Maestra de Colombia).

"La formación de los profesores es determinante. La inclusión la hace el docente. El profesor tiene que estar concienciado de las necesidades de los alumnos y tiene que incluir a los alumnos con todos los demás. Tiene que hacerlo uno más de la clase. No considerarlo que es el alumno de la profesora de apoyo. Tiene que trabajar con él desde el primer momento" (Profesora especialista en alumnos con necesidades. España).

\subsubsection{Hipótesis 2. "Los maestros considerarán} que los alumnos con necesidades educativas especiales asociadas a discapacidad tienen dificultades de relación con su grupo de iguales”

El 40,6 \% de los maestros encuestados en los tres países consideran que los alumnos con necesidades educativas especiales asociadas a discapacidad presentan dificultades para establecer relaciones sociales. Sin embargo, existen diferencias entre las respuestas recogidas en los tres países.

\section{Figura 2}

Porcentaje de maestros que considera que "los alumnos con necesidades educativas especiales asociadas a discapacidad suelen tener dificultades en las relaciones sociales"

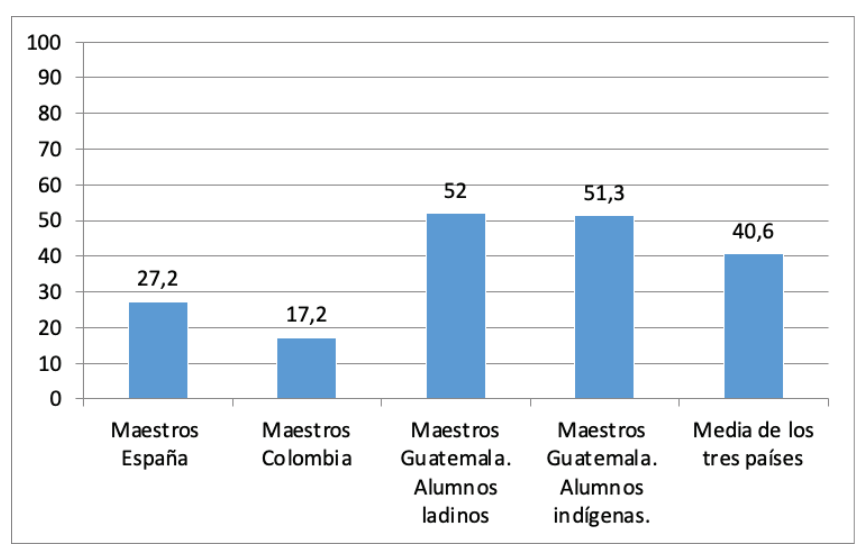

Como puede observarse en el gráfico 2, los maestros de escuelas guatemaltecas (tanto los maestros de zonas indígenas como ladinas) son los que más afirman que los alumnos con necesidades educativas especiales tienen dificultades para establecer relaciones sociales $(51,35 \%$ y $52 \%$, respectivamente). No obstante, entre los testimonios recopilados en Guatemala se encuentras dificultades, pero también logros:

"Con sus compañeros es algo difícil porque no

la entienden a veces. Hemos avanzado en que la respeten más, pero no hemos logrado el $100 \%$. (Maestra de Guatemala).

"Los compañeros los incluyen sin problema. Ayudan a la maestra. Tienen una actitud de mucho respeto. (Directora de Guatemala).

En Colombia, solo el 17,24\% de los maestros encuestados considera que los alumnos con necesidades educativas especiales tienen dificultades en establecer relaciones sociales, frente al 27,2 \% de los maestros españoles.

En las entrevistas realizadas a profesores de España se indica cómo en algunas ocasiones los niños con necesidades educativas especiales tienen mayores dificultades en las relaciones con su grupo de iguales por no compartir el mismo tipo de intereses o por 
dificultades de acceso. Según aumenta la edad de los alumnos, tienen mayores problemas en la interacción de los niños con el compañero con discapacidad, especialmente en el momento de jugar. Se identifica que estas dificultades son más marcadas en el caso de los niños con discapacidad intelectual.

"Son muy aceptados por la clase en general, aunque a la hora de jugar no juegan todos con él porque tienen otros tipos de intereses. Ellos mismos se van agrupando, los nińos con discapacidad hacen grupo.” (Maestro de España).

"Se percibe más aislamiento en niños mayores porque el juego va evolucionando. En infantil son juegos más cooperativos (parque infantil, palas, etc.) y posteriormente en primaria es un juego más individualizado, les cambia los intereses." (Maestra de España).

"Conforme se van haciendo mayores, es más complicado incluir a los niños con necesidades educativas especiales" (Maestro de España).

"Con niños con discapacidad intelectual sí empiezan a marcar la diferencia antes porque no tienen los mismos intereses" (Maestra de España).

"Con los niños con discapacidad motora lo intentan, pero es verdad que la barrera de la silla no les permite jugar con ellos igual que con el resto. No pueden jugar al baloncesto, no pueden jugar al futbol. Aunque estén incluidos en su aula de referencia, en el momento de realizar muchas actividades no pueden hacerlas con ellos. No es porque no quieran acercarse a ellos o no los valoren, sino porque hay una barrera arquitectónica, una silla de hierro con la que chocan" (Maestro de España).

No obstante, los diferentes testimonios reflejan cómo los alumnos acogen a sus compañeros con necesidades educativas especiales:

"Los niños de necesidades educativas especiales no tienen problemas en infantil ni primaria. En primaria los compañeros son "tutores" y les ayudan. En infantil, si hay algún niño con discapacidad, se actúa desde el principio con normalidad" (Equipo directivo de colegio de España). "Es increíble cómo los compañeros acogen los niños con necesidades educativas especiales. Los niños nos ayudan a nosotros. Una norma de la clase es ayudarles" (Maestra de España).

"Los niños son muy inclusivos, los acogen, incluso algunas veces en exceso y hay una sobreprotección de su grupo de iguales a los niños con necesidades. Están muy encima de ellos tanto en las clases como en el recreo" (Maestra. España).

\subsubsection{Hipótesis 3. "Los maestros valoran posi-} tivamente la inclusión de los alumnos de otras culturas. Los países con mayor jerarquía cultural (Colombia y Guatemala) mostrarán menos apoyo"

Con relación a la inclusión de los alumnos de otras culturas, los maestros tienen opiniones más positivas que con los alumnos con discapacidad. El 72,4\% de los maestros encuestados en los tres países considera que los alumnos de otras culturas aprenden más si asisten a escuelas ordinarias. El 27,6 \% de los maestros encuestados considera lo contrario.

\section{Figura 3}

Porcentaje de maestros que considera que "los alumnos de otras culturas aprenden más si asisten a escuelas multiculturales"

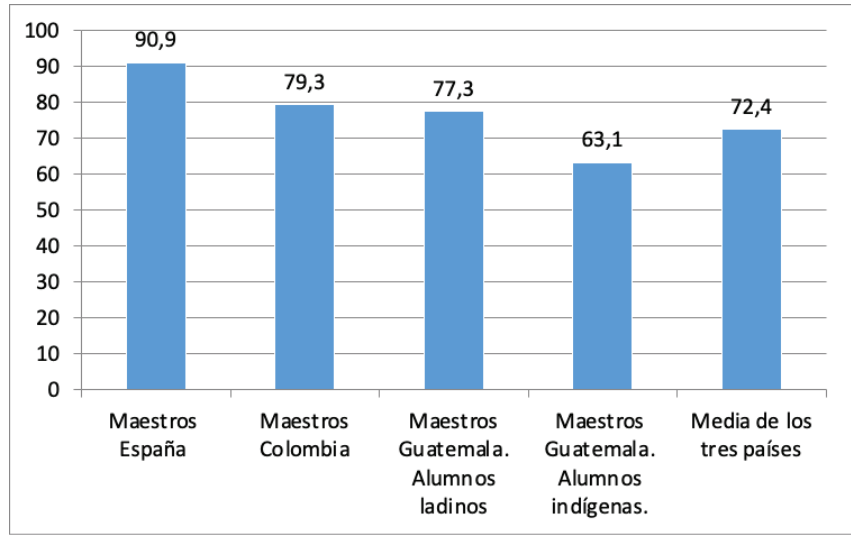

c. Entendiéndose como escuelas multiculturales centros educativos con alumnos de diversas culturas juntos. 
Cómo puede observarse en el gráfico 3, los maestros españoles son los que más valoran que los alumnos de otras culturas aprenden más si asisten a escuelas normales. El 90,90 \% responden de manera positiva a esta pegunta. Ello concuerda con las opiniones recogidas en las entrevistas en las que la mayoría de los profesores españoles reflejaron los beneficios de la presencia de niños procedentes de otras culturas y países.

"Para nosotros es habitual tener en la escuela alumnos de diferentes grupos culturales. No hacemos ningún tipo de distinción. La diversidad es muy positiva. Es muy enriquecedor para todos. Se benefician todos los niños." (Maestro de España).

"Que tengamos niños que proceden de otros países es un aspecto bueno, nos enriquece. A los nińos les decimos que los nuevos nos van a entregar lo que traen y nosotros le devolvemos lo que tenemos aquí. Para nosotros es una alegría que haya diferencias" (Maestro de España).

"Es fantástico. Saben cantar cumpleaños feliz en los diferentes idiomas: en árabe, chino, español etc. En las fiestas cada uno trae las cosas típicas de sus países, hacemos semanas culturales, leemos cuentos de los diferentes países, la bienvenida está en diferentes idiomas. Es nuestra señal de identidad" (Maestro de España).

En los países con mayor jerarquía cultural, Colombia y Guatemala, los maestros consideran que es algo más difícil que los alumnos afrodescendientes e indígenas aprendan juntos a los compañeros de la cultura dominante o alumnos blancos, especialmente en el caso de los alumnos indígenas en Guatemala.

Las opiniones de los maestros de las escuelas de Guatemala con alto porcentaje de alumnos indígenas reflejan que el 63,15\% considera que los alumnos de otras culturas aprenden más en escuelas ordinarias, es decir centros educativos en los que estudian alumnos de diferentes culturas. Ello conlleva que el 36,85\% de los maestros de localidades indígenas considere que los alumnos aprenderían más en escuelas específicas, como las interculturales bilingües, en las que estudian alumnos indígenas con compañeros indígenas. Este porcentaje es algo menor (22,7\%) en los maestros de Guatemala de escuelas de zonas ladinas, que no tienen entre sus alumnos niños/as indígenas.

Esto puede estar justificado, como indican los testimonios recopilados en Guatemala, por la existencia de asimetrías en el acceso al desarrollo y a una situación de exclusión, al comparar la estructura socioeconómica de las poblaciones indígenas y no indígenas.

"La situación de la mayoría de la población indígena es crítica. Este sector presenta indicadores de desarrollo bastante por debajo de la población en general, especialmente de la población ladina. Altos porcentajes de analfabetismo, de fracaso escolar, de falta de asistencia en salud, de desnutrición crónica infantil, entre otros. Es el sector que más sufre los efectos del racismo y la discriminación. Hoy por hoy, la población indígena del país presenta los peores indicadores de desarrollo con relación al resto de los pueblos que conforman el país, principalmente frente a la población ladina” (Especialista Educación, Guatemala).

"La educación escolar se hace en español, se valora más la cultura ladina”. (Especialista Educación. Guatemala).

"En las escuelas que están dentro de los pueblos no se da mucho esta discriminación porque la mayoría de la población es indígena. Los maestros y los directores son indígenas. Aquí, por ejemplo, en Comalapa hay pocas personas ladinas y no se da esa discriminación. Es mas en la zona urbana. Si algún estudiante se va a la capital, a pesar de ser del mismo país, va a sufrir discriminación” (Coordinadora del Programa Hogares Comunitarios. Guatemala).

"Hay muy poca discriminación en el pueblo. Como es una comunidad indígena, las personas se sienten orgullosas de ser indígenas. Pero otra cosa es cuando salen del pueblo" (Escuela Guatemala).

Como se refleja en las entrevistas, un porcentaje significativo de maestros consideren que los alumnos de otras culturas no aprenden más en escuelas ordinarias puede ser también debido a las limitaciones a las que se enfrentan para ajustar la respuesta educativa a las necesidades de las comunidades indígenas y 
a la gran diversidad cultural y lingüística presente en el país. Existen dificultades en la implementación de una educación intercultural bilingüe de calidad por factores como la falta de formación del profesorado o la falta de incorporación en el currículo de la diversidad cultural del país:

"El currículum tiene una carencia de la historia cultural de los distintos pueblos. No hay que dejar de lado el castellano, pero tampoco la parte filosófica, matemáticas, historia etc. de los mayas. Muchas veces se rechaza porque no se conoce la cultura desde el pueblo" (Especialista en educación, Guatemala).

"Hay comunidades en las que los niños en una misma aula hablan tres idiomas más el castellano. Hay maestros que no tienen la capacidad de atender a estos niños con más de dos o tres idiomas. Al docente no se le han dado las herramientas de cómo jugar y como trabajar con alumnos de diferentes características en el aula. Como el docente trabaja en castellano y no conoce la cultura del niño, no le puede dar respuesta. Se ha trabajado en el currículum la información de los pueblos maya, xinca, garífuna y ladinos, pero de manera aislada y puntual” (Directora de escuela, Guatemala).

"Hay una tensión en los estudiantes, maestros y padres. Muchos padres no aceptan el idioma maya en la escuela por toda la trayectoria de discriminación que ha tenido. Muchos maestros porque no saben cómo trabajar en el área bilingüe, unos porque no hablan y otros porque no quieren. Muchas veces el problema es que no tienen las herramientas para trabajar. Se implementa idioma, pero no se les da a los maestros las herramientas. Esto genera una gran tensión en las zonas multilingües en las que hay aulas con niños que hablan varios idiomas" (Especialista Educación Intercultural Bilingüe. Guatemala).

"Las profesoras son ladinas y no hablan maya, lo que hace que los niños indígenas no puedan hablar su lengua. Es una gran carencia. Hay veces que tienen dificultades en el lenguaje porque tienen dificultades en el castellano. El año pasado tuvimos un niño que no hablaba español. Lo pasó mal y para él fue muy difícil la escuela” (Maestra de Guatemala).

Finalmente, el 79,3\% de los profesores colombianos de alumnos afrodescendientes considera que los alumnos de otras culturas aprenden más en escuelas ordinarias, lo que implica que el 20,7 \% de los profesores considera que aprenden más en otro tipo de escuelas (los porcentajes son muy similares a los maestros encuestados de Guatemala de escuelas sin alumnos indígenas).

$\mathrm{Al}$ igual que en Guatemala, diferentes testimonios en Colombia reflejan la importancia de la escuela en la transmisión de los valores que posteriormente determinarán las actitudes de los profesores. El currículo, los materiales educativos o las actitudes y opiniones de los maestros son elementos fundamentales en la escuela para avanzar en creencias y comportamientos más equitativos e inclusivos.

"Los estereotipos se encuentran en el currículum y en los materiales educativos. Si uno realizara un análisis juicioso de los cuentos y los textos que los niños reciben, va a encontrar muchos estereotipos incluidos". (Maestro de Colombia).

"Para generar nuevos imaginarios en los niños y niñas son necesarios referentes de éxito dentro de la población afrodescendiente. Deportistas, cantantes y científicos están apareciendo en los últimos años, pero necesitamos referentes médicos, políticos, escritores. Es importante que las personas negras sean parte de la vida cotidiana de un país. Hoy es una excepción tanto para nosotros como para los indígenas" (Especialista en Educación, Colombia).

\subsubsection{Hipótesis 4. "Los maestros considerarán} que los alumnos de otras culturas no tienen dificultades en las relaciones sociales"

Los datos obtenidos reflejan que el 31,9\% de los maestros encuestados en los tres países cree que los alumnos de otras culturas suelen tener dificultades en las relaciones sociales. 


\section{Figura 4}

Porcentaje de maestros que considera que "Los alumnos de otras culturas suelen tener dificultades en las relaciones sociales"

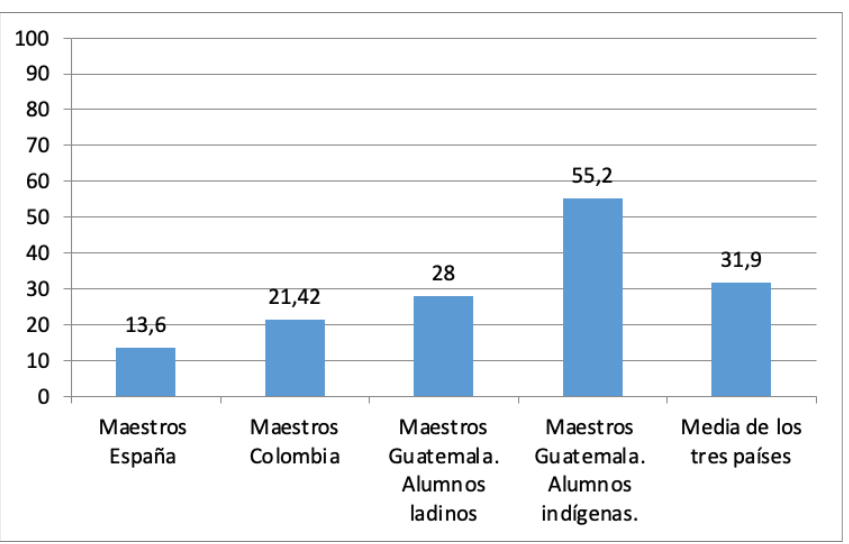

Los datos de la figura 4, reflejan la misma tendencia que en la hipótesis anterior. En el caso de los maestros espańoles, el 86,37\% considera que los alumnos de otras culturas no tienen dificultades para establecer relaciones sociales, es decir solo el $13,6 \%$ de profesores considera que si tienen dificultades de relación con el grupo de iguales.

En el caso específico de alumnos inmigrantes en España es importante tener presentes dos variables, no analizadas de manera directa en el estudio, que influyen en los vínculos y relaciones sociales: la existencia o no de una lengua común con los compañeros; y el nivel sociocultural y económico de la región en la que se encuentre la escuela. Respecto al primer punto existe diferencia entre los alumnos inmigrantes hablantes de castellano y los alumnos inmigrantes procedentes de otros países que hablan otra lengua. Estos últimos tienen más dificultades de relación social, especialmente en los primeros momentos de llegada al país. Respecto a la segunda variable, como se indica en uno de los testimonios, hay contextos socioculturales en los que las familias "autóctonas” están más sensibilizadas a favor de la inclusión de personas con otras culturas.

"En colegios en donde hay mucho tipo de alumnos

y muchas diferencias sociales, la participación y colaboración entre los niños está muy presente. Por ejemplo, en el juego no hacen muchas distinciones. Siempre hay algún caso excepcional, pero los niños están muy concienciados con las diferencias. Lo ven muy normal, no ven problema. Quizás en colegios con nivel sociocultural más elevado, en el que las familias no tienen tanta relación con las minorías étnicas, haya más dificultad, pero esta se produce por desconocimiento por parte de los maestros y las familias" (Maestra de España).

En Guatemala, el 55,26 \% de los maestros de alumnos indígenas considera que los alumnos de otras culturas suelen tener dificultades para establecer relaciones sociales, un dato que está en consonancia con las dificultades expresadas en la pregunta anterior. Este porcentaje es menor en las respuestas de los maestros de Guatemala de alumnos ladinos, de los cuales el 28 $\%$ considera que tienen dificultades sociales. Estas dificultades que perciben los maestros también pueden ser causadas por el uso de una lengua diferente, que obstaculiza la relación con los compañeros que no son indígenas. Además, es muy posible que la distancia en la valoración cultural y social entre ladinos (blancos) e indígenas sea la razón principal de la dificultad de las relaciones sociales.

A pesar de estos problemas, existe un deseo de los niños indígenas de jugar y estudiar con compañeros blancos antes que elegir a los de su propio grupo cultural, posiblemente para mejorar su estatus social (Hernández, 2015). Lo que puede suceder -y así se comprueba en este estudio- es que los niños blancos no eligen a los indígenas para jugar y estudiar con ellos. Posiblemente sea esta la variable principal que influye en las opiniones de los maestros de alumnos indígenas.

Algunos de los testimonios obtenidos confirman estas interpretaciones.

"Yo no sabía español y sufrí mucho en la escuela cuando fui a estudiar a Antigua. Si hubiera ido a algún pueblo no hubiera sufrido tanto pero mi papá se tuvo que desplazar por trabajo. Al final aprendí español, pero me costó mucho. Mis compañeros se reían de mí cuando mezclaba el Cachiquel con el espańol" (Maestra. Centro Cultural Maya Bilingüe, Guatemala).

"Cuando yo fui a la ciudad, por el trabajo de mi papá, fue cuando yo sufrí discriminación. Yo usaba el traje, pero me aislaban mucho en el recreo, no 
jugaban mis compañeros conmigo. También lo sufrí de las maestras. Me afectaba mucho y me cambiaron a otro colegio después de 3 años. Para mí fue muy marcado porque yo venía del pueblo" (Maestra, Guatemala).

"El ideal de la mayoría de la población indígena es ser como el ladino, tener todos los beneficios que los ladinos tienen. Estas ideas son sembradas en los niños y de ahí el deseo de jugar y estudiar con compañeros blancos en lugar de los de su cultura" (Especialista de Educación, Guatemala).

"Que los niños indígenas prefieran juntarse con los blancos es consecuencia de la misma historia social de los países latinoamericanos. En la estratificación social blancos-criollos estaban por encima de negros-mulatos e indígenas. Esto se ha transmitido generación tras generación.” (Especialista en afrodescendientes, Colombia).

Si se analizan los datos de Colombia, el 21,42\% de profesores considera que los alumnos de otras culturas tienen dificultades de relación con el grupo de iguales. Nuevamente los datos (porcentaje inferior a Guatemala, pero superior a España) son reflejo de la estratificación de la población y los prejuicios que se inculca a los alumnos desde que son pequeños y que conlleva la elección de amigos y compañeros blancos frente a niños de otras culturas.

"Hasta los 6 años, los niños están en pleno proceso de aprendizaje y descubrimiento. Y lo que aprecian es que la sociedad mayoritaria tiende a ser blanca o a adoptar modales blanco-europeos. Descubren los prejuicios sobre su grupo y en un intento de huir de esta condición, lo más cercano que encuentran es al niño blanco con quien se unen. A los 8 años lo habitual es que los niños afrodescendientes mantengan su relación con los blancos, condicionados por el imaginario social dominante. Aunque, sin embargo, hay niños que empiezan a cuestionar el modelo social en el que viven, porque van teniendo conciencia de la discriminación que sufre su grupo. Y esto refuerza sus vínculos con otros miembros de su comunidad, con el objetivo de protegerse y para reivindicar su condición humana en igualdad con los demás. (Especialista en afrodescendientes, Colombia).

\section{Conclusiones}

Los tres países implicados en el estudio, Colombia, Guatemala y España; han incluido en su legislación la importancia del acceso y permanencia de los alumnos con necesidades educativas especiales asociadas a discapacidad o de diferentes culturas en las escuelas. Sin embargo, la realidad es muy diferente en función de la situación social y educativa de cada uno de los países.

Respecto a los alumnos con necesidades educativas especiales en España, después de 35 años desde el inicio sistemático de políticas y prácticas inclusivas, los datos obtenidos muestran respuestas ambivalentes. El 42,9 \% de los maestros españoles encuestados consideran que los alumnos con discapacidad aprenden menos en escuelas ordinarias. Este dato indica que casi la mitad de los maestros consultados valora que no existen las condiciones adecuadas para conseguir una inclusión plena de los alumnos con necesidades educativas especiales asociadas a discapacidad en la escuela ordinaria.

A su vez, el 27,2\% reflejan que los alumnos con necesidades educativas especiales tienen dificultades en las relaciones sociales con los compañeros.

En Guatemala, con políticas y prácticas inclusivas más incipientes y con condiciones sociales y educativas más desfavorables, se percibe una actitud más negativa en la sociedad y en la escuela. El proceso de inclusión comenzó en el año 2000, y muestra en la actualidad bastantes dificultades en el acceso y permanencia de los niños con discapacidad en el sistema educativo. Solo el 46,8\% de los maestros de Guatemala consideran que los alumnos con discapacidad aprenden más en escuelas ordinarias, y el 51,65\% manifiestan que estos alumnos tienen dificultades de relación social con los compañeros. También, se identifican dificultades en la inclusión y se resalta la falta de formación de los profesionales que promuevan un proceso de enseñanza y aprendizaje en que los alumnos con necesidades educativas especiales, asociadas a discapacidad, adquieren las competencias necesarias para lograr su autonomía e inclusión social en la etapa adulta. Dicho aspecto concuerda con lo indicado por Rea (2011) al hacer referencia sobre los resultados positivos de la 
formación del profesorado en materia de educación inclusiva.

En Colombia, a diferencia de lo inicialmente esperado en el estudio (que los maestros que trabajan en países con menos recursos mostrarían más reservas a la inclusión de alumnos con necesidades educativas especiales que los españoles), el 64,2\% de los maestros consideró que los alumnos con discapacidad aprenden más en escuelas ordinarias y solo el 17,24\% indicó que los alumnos con discapacidad tienen dificultades en establecer relaciones sociales.

Respecto a la inclusión de alumnos de diferentes culturas, las respuestas de los profesores de las escuelas de España muestran una actitud positiva hacia la incorporación de alumnos inmigrantes en las escuelas. El 90 $\%$ de los maestros espańoles encuestados considera que los alumnos de otras culturas aprenden más si asisten a escuelas multiculturales (escuelas en las que hay alumnos de diferentes culturas juntos) y solo el 13,6 \% de ellos considera que los alumnos de otras culturas tienen dificultades de relación con sus compañeros.

En Colombia y Guatemala, en cambio, se identifican grandes barreras actitudinales y de discriminación hacia la población indígena y afrodescendiente, que son transmitidas a los más pequeños desde su contexto educativo, familiar y social.

Los datos cuantitativos obtenidos en el cuestionario a los profesores de Guatemala que trabajan con alumnos indígenas ponen de manifiesto que el $63.1 \%$ considera que los alumnos de otras culturas aprenden más si asisten a escuelas multiculturales, lo que supone que el $36,9 \%$ de los maestros de alumnos indígenas prefieren que sus alumnos estudien sin mezclarse con los ladinos para evitar posibles experiencias de discriminación.

Así mismo, el 55,2 \% de los maestros de zonas indígenas indica que los alumnos de otras culturas tienen dificultades en las relaciones sociales con sus compañeros. Estas creencias proceden de la constatación de que los alumnos de la cultura "dominante" tienden a excluir a sus compańeros indígenas, lo que es una reproducción de las relaciones establecidas en la sociedad y en la cultura del país.
Para interpretar las respuestas en Guatemala es necesario diferencias entre las escuelas ordinarias, es decir, aquellas en las que los alumnos de diferentes culturas estudian juntos, sin establecer distinción de las escuelas bilingües. Cómo se indicó en el marco teórico, estas últimas tienen un enfoque alternativo de aprendizaje para los nińos y niñas indígenas hablantes del idioma maya ixil inicialmente, extendiéndose más tarde a los idiomas k'iche', kaqchikel, q'eqchi' y mam. Ello conllevó a la creación de escuelas interculturales bilingües que dan respuesta educativa a los niños y niñas indígenas en edad escolar de las comunidades mayas, que tienen mayor número de hablantes de los dichos idiomas, de preprimaria a $6^{\circ}$ grado de primaria.

La influencia de los medios de comunicación, la sociedad, la historia y la escuela son factores determinantes de la mayor valorización de la cultura ladina frente a la indígena. No obstante, hay que indicar que en zonas rurales y pueblos en los que la mayoría de la población es indígena, estas comunidades no sienten tanta discriminación como en zonas urbanas

En Colombia, el $79.3 \%$ considera que los alumnos de otras culturas aprenden más si asisten a escuelas multiculturales y el 21,42\% indica que los alumnos de otras culturas tienen dificultades en las relaciones sociales. Los datos, aunque más moderados que en Guatemala, son reflejo también de la estratificación de la población y de los prejuicios que se inculca a los alumnos desde que son pequeños.

Finalmente, es importante destacar que las respuestas de los entrevistados señalan la importancia de un cambio en las actitudes sociales que facilite una educación intercultural de calidad. Es preciso, pues, que se refuerce el conocimiento y la valoración de las diferentes culturas, así como la formación del profesorado para que este pueda dar una respuesta adecuada a la educación de todos sus alumnos. Una valoración que depende en gran medida de un mayor reconocimiento y de una progresiva igualdad en la sociedad de los diferentes grupos culturales. De esta forma, será posible romper la transmisión de valores de discriminación en los alumnos. 
El análisis y la interpretación de la información obtenida revela que existen luces y sombras hacia la inclusión educativa y social de los diferentes colectivos. A pesar de los logros alcanzados en las últimas décadas, tras el análisis de los diferentes relatos se puede observar que los diferentes grupos estudiados, indígenas, afrodescendientes y personas con discapacidad, presentan situaciones de rechazo, discriminación y/o segregación educativa y social por su condición personal o cultural.

La comparación de las informaciones obtenidas en los tres países indica que los factores determinantes de esta discriminación son las desigualdades sociales y culturales, así como las condiciones educativas que existen en cada país: cuanto mayor es la desigualdad y peores son las condiciones educativas, más difícil es la inclusión educativa.

\section{Referencias}

Ainscow, M. (6 septiembre de 2017). Pasos para la inclusión en las escuelas https://cutt.ly/8h7Ugg0

Abbott, L. (2006). Northern Ireland headteachers' perceptions of inclusion. International Journal of Inclusive Education, (10), 627-643.

Avramidis, E. y Norwich, B. (2002). Teachers' attitudes towards integration/inclusion: a review of the literature. European Journal of Special Needs Education, (17), 129-147.

Boer, B. (2012). Inclusion: a question of attitudes? A study on those directly involved in the primary education of studens with special educational needs and their social participation. Groninger: Stichting Kinderstudies.

Calderón, F., Hopenhayn, M. y Ottone, E. (1996). Esa esquiva modernidad: desarrollo, ciudadania y cultura en América Latina y el Caribe. UNESCO-Nueva Sociedad.

Carbonell, F. (2002). Educación intercultural: principales retos y requisitos indispensables. Cooperación Educativa, (65), 63-68.

Consejo Nacional para la Atención de las Personas con Discapacidad (2006). Politica nacional en discapacidad. Guatemala: CONADI.
Echeita, G. (2017). Educación inclusiva. Sonrisas y lágrimas. Aula Abierta, (46), 17-24.

Fernández, M. y Terrén, E. (2008). De inmigrantes a minorías: temas y problemas de la multiculturalidad. Revista de educación, (345), 15-21

Florian, L. (2014). Reimagining special education: why new approaches are needed. En L. Florian. The SAGE handbook of special education. (pp. 9-22). SAGE.

Gómez Beltrán, J. C. (2010). Discapacidad en Colombia: Reto para la Inclusión en Capital Humano. Bogotá: Fundación Saldarriaga Concha.

Hernández, L. (2015). Estudio sobre las actitudes de los niños de 4 a 8 años hacia las diferencias étnico-culturales y hacia la discapacidad en Colombia, Guatemala y España. [Tesis doctoral, Universidad Complutense de Madrid]. https:// eprints.ucm.es/34359/

López, N. (coord.) (2012). Equidad educativa y diversidad cultural en América Latina. Buenos Aires: IIPE/UNESCO.

Marchesi, A., Tedesco, J. y Coll, C. (coord.) (2011). Calidad, equidad y reformas en la enseñanza. Madrid: OEI/Fundación Santillana.

Marchesi, A. y Martín, E. (2014). Calidad de la enseñanza en tiempos de crisis. Madrid: Alianza Editorial.

Marchesi, A. Coll, C. y Palacios, J. (2017). Desarrollo psicológico y educación: respuestas educativas a las dificultades de aprendizaje y del desarrollo. Madrid: Alianza Editorial.

Marshall, J., Ralph, S., y Palmer, S. (2002). 'I wasn't trained to work with them': mainstream teachers' attitudes to children with speech and language difficulties. International Journal of Inclusive Education, (6), 199-215.

Meijer, C. J. W. (2003). Integración en Europa: disposiciones relativas a alumnos con necesidades especiales. Tendencias en 14 países europeos. Real Patronato de Prevención y de Atención a Personas con Minusvalías. 
Ministerio de Educación y Formación Profesional (2018). Estadisticas enseñanzas no universitarias. Alumnado con necesidad especifica de apoyo educativo. Instituto Nacional de Estadística.

https://cutt.ly/dh7Ur1J

Pearman, E. L., Huang, A. M. y Mellblom, C. I. (1997). The inclusion of all students: concerns and incentives of educators. Education and Training in Mental Retardation and Developmental Disabilities, 32(1), 11-20.

Rahona, M. y Morales, S. (2013). Educación e inmigración en España: desafios y oportunidades. Colección Metas Educativas. OEI-Fundación Alternativas. https://cutt.ly/Ph7UiTU

Rea, H., McKenzie, K., y Murray, G. (2011). The impact of training on teacher knowledge about children with an intellectual disability. Journal of Intellectual Disability, 15(1), 21-30.
Roux, G. (2010). Politicas públicas para el avance de la población afrocolombiana: revisión y análisis. Panamá: Programa de las Naciones Unidas para el Desarrollo.

Sales, A., Moliner, O. y Sanchiz, M. L., (2001). Actitudes hacia la atención a la diversidad en la formación inicial del profesorado. Revista Electrónica Interuniversitaria de Formación del Profesorado, 4(2).

Zapata, R. (2014). Diversidad cultural en la formación del futuro profesorado en América Latina. Necesidades y perspectivas. Revista Electrónica Interuniversitaria de Formación del Profesorado, 17(2), 219-234. 